(C) 2001 International Press

Adv. Theor. Math. Phys. 5 (2001) 139-163

\title{
Lagrangians for the
}

\section{Gopakumar-Vafa conjecture}

\author{
Clifford Henry Taubes ${ }^{1}$ \\ Department of Mathematics \\ Harvard University \\ Cambridge, MA 02138 \\ chtaubes@math.harvard.edu
}

Gopakumar and Vafa [GV] have have conjectured the existence of a fundamental relationship between Gromov-Witten type invariants of holomorphic curves in the vector bundle $O(-1) \oplus O(-1)$ over $\mathbb{P}^{1}$ and certain knot invariants, for example the Jones polynomial. They came to their conjecture by applying a fundamental observation of 't Hooft $[\mathrm{H}]$ in a string theoretic context on $T^{*} S^{3}$ described by Witten [W]. Subsequently, the scope of the conjecture was expanded by Ooguri and Vafa [OV]. Successful tests of the have been made, for example, by Labastida and Marino [LM], Ramadevi and Sarkar [RS], Labastida, Marino and Vafa [LMV] and Aganagic, Klemm and Vafa [AKV]. In the mean time, Faber and Pandarhapande [FP], Katz and Liu [KL] and Li and Song [LS] have considered the mathematical foundations for the conjecture and verified certain parts of it.

\footnotetext{
${ }^{1}$ Supported in part by the National Science Foundation

e-print archive: http://xxx.lanl.gov/math-ph/0201219
} 
The verification of Gopakumar and Vafa's proposal has been slow, in part because the string theoretic arguments have not provided a geometric correspondence between a particular knot and a particular set of holomorphic curves in $O(-1) \oplus O(-1)$. Even so, it is a good bet, verified in part by Katz and Liu [KL], Labastida, Marino and Vafa [LMV] and Aganagic, Klemm and Vafa [AKV], that such a correspondence exists and that it is mediated by a suitable Lagrangian 3-manifold sitting in $O(-1) \oplus O(-1)$. To be specific, the knot should determine the Lagrangian, and then a knot invariant should come as a suitable count of compact, holomorphic curves with boundary on the Lagrangian.

This said, the mathematics of counting holomorphic curves with boundary on a Lagrangian submanifold dates back to Floer's original work on the Arnold conjecture $[\mathrm{F}]$. Moreover, since Floer's work, such counts have been considered by mathematicians in myriad circumstances. Yet, each new circumstance typically has new technical problems to surmount; and in this regard, Katz and Liu [KL] found the story here to be typical.

Curve counting theory aside, the proposed mechanism via Lagrangians for Gopakumar and Vafa's conjecture requires knots to provide Lagrangians in $O(-1) \oplus O(-1)$. This article addresses the latter concern. By way of preliminary remarks, note that the construction described below produces a 2-dimensional Lagrangian surface in $\mathbb{C}^{2}$ from a knot in $S^{3}$, designed so that the Lagrangian surface intersects all large radius 3-spheres as an isotopy of the knot. Moreover, if the starting knot is isotopic to one that is mapped to itself via $S^{3}$ 's antipodal map, then the resulting Lagrangian is used to construct a 3-dimensional Lagrangian in $O(-1) \oplus O(-1)$ that fibers over the equator in $\mathbb{P}^{1}$ with the 2 -dimensional Lagrangian as fiber. Thus, the construction below can be viewed as one that constructs a Lagrangian in $O(-1) \oplus O(-1)$ from a knot in $\mathbb{R}^{3}$.

By the way, start with a knot in the unit radius sphere in $S^{3}$ and Gromov's $h$-prinicple [G] more or less asserts that there is a Lagrangian surface in the unit ball of $\mathbb{C}^{2}$ that intersects the boundary 3 -sphere as the given knot. This said, the construction below provides explicit Lagrangians. In particular, a realization of the knot as a braid provides a Lagrangian whose topology can be read off directly from the properties of the braid.

Here is how the remainder of this article is organized: The first section describes the construction of Lagrangians in $\mathbb{C}^{2}$ from braids in $S^{3}$ that intersect all large radius 3 -spheres as a braid that is braid isotopic to the original. The initial steps construct immersed disks, and it is then explained how the immersion points can be smoothed to produce embedded, although not always 
orientable, Lagrangian surfaces. Section 2 explains how the double points of the immersed disks relate to the crossings of a certain projection of the original braid. The third section relates the topology of three Lagrangians coming from a triad of braids that arise in standard discussions of skein relations. Section 4 constructs Lagrangian 3-manifolds in $O(-1) \oplus O(-1)$ from certain Lagrangian surfaces in $\mathbb{C}^{2}$, and the final section views these $O(-1) \oplus O(-1)$ Lagrangians from a dual perspective on $T^{*} S^{3}$. Note that the discussion that follows owes much to conversations between the author and Professor Cumrun Vafa to whom thanks is offered.

\section{The construction of Lagrangians}

My purpose here This section is to ddescribes a construction that starts with a connected, $N$-stranded braid in $S^{3}$ and constructs of an a properly immersed, Lagrangian disk in $\mathbb{C}^{2}$ that intersects large radiiall sufficiently large radius 3 -spheres in $\mathbb{C}^{2}$ as an $N$-stranded braid that is braid isotopic to the original. The construction is then generalized to obtain immersed Lagrangians for braids with more than one component, and then generalized again to provide embedded (but possibly non-orientable) Lagrangians. The construction is divided into ten steps. 3 -spheres as a given $N$-stranded braid. The construction is generalized at the end to the case of links.

Step 1: To start the construction, introduce $\left(z_{1}, z_{2}\right)$ to denote the standard, complex coordinates on $\mathbb{C}^{2}$, defined so that the symplectic form is given as

$$
\omega \equiv i 2^{-1}\left(d z 1 \wedge d \bar{z}_{1}+d z_{2} \wedge d \bar{z}_{2}\right) .
$$

Next, introduce the 'hyperkähler' rotated complex coordinates

$$
a 1=2^{-1 / 2}\left(z_{1}-\bar{z}_{2}\right) \text { and } a_{2}=2^{-1 / 2}\left(z_{2}+\bar{z}_{1}\right)
$$

with respect to which

$$
\omega=i 2^{-1}\left(d a_{1} \wedge d a_{2}-d \bar{a}_{1} \wedge d \bar{a}_{2}\right) .
$$

Since this rotation is orthogonal, the metric in the $\left(a_{1}, a_{2}\right)$ coordinates is the standard one. Note that the $a_{1}$-plane is a Lagrangian plane in $\mathbb{C}^{2}$ with respect to $\omega$. Of course, so is any surface given as the zeros of a function of $\left(a_{1}, a_{2}\right)$ that is holomorphic.

In the subsequent steps, the complex coordinate $a_{1}$ is written in terms of its real and imaginary parts as $a_{1}=x^{1}+i x^{2}$. At the same time the 
coordinate $a_{2}$ is written somewhat perversely as $a_{2}=p_{2}+i p_{1}$. In terms of these real coordinates,

$$
\omega=d p_{1} \wedge d x_{1}+d p_{2} \wedge d x^{2} .
$$

The choice of coordinates here is meant to stress an implicit identification below between the $a_{2}$-direction in $\mathbb{C}^{2}$ and the fiber of the cotangent bundle of the $a_{1}$-plane. More to the point, this gives a symplectic identification between $\mathbb{C}^{2}$ and the cotangent bundle, $T^{*} \mathbb{C}$, of the $a_{1}$-plane.

The reason for making such an identification is as follows: If $f$ is any smooth, locally defined function of the coordinates $\left(x_{1}, x_{2}\right)$, then the graph of $d f$ defines a Lagrangian surface in $T^{*} \mathbb{C}$ and hence in $\mathbb{C}^{2}$. To be explicit, the locus of points in $T^{*} \mathbb{C}$ where $\left(x^{1}, x^{2}, p_{1}=\partial_{1} f, p_{2}=\partial_{2} f\right)$ is a Lagrangian surface.

Step 2: Fix attention on some given connected, $N$-stranded braid $K$. To be more precise about what this means here, identify $S^{1}$ with the unit circle in $\mathbb{C}$ with coordinate $\zeta$ such that $|\zeta|=1$. This done, then $K$ can be viewed as an embedded circle in $S^{1} \times \mathbb{C} \subset \mathbb{C}^{2}$ obtained as the image of a map from $S^{1}$. In particular, such a map should send $\eta \in S^{1}$ to $\left(\zeta=\eta^{N}, z=\gamma(\eta)\right)$ with $\gamma$ a map to $\mathbb{C}$ that separates pairs of points in $S^{1}$ whose ratio is a non-trivial, $N^{\prime}$ th root of unity. In this regard, it proves convenient below to write $\eta=e^{i \theta / N}$ with $\theta \in[0,2 \pi N]$. Thus, $\gamma$ is a function of $\theta$ that is periodic with period $2 \pi N$ and $\zeta=e^{i \theta}$ is a function of $\theta$ with period $2 \pi$. This notation identifies the unit circle in the $a_{1}$-plane to the interval $[0,2 \pi]$ with its endpoints identified.

Note that the graph of any $2 \pi N$ periodic complex function, $\gamma$, of $\theta$ defines a braid as long as the $N$ values $\{\gamma(\theta+2 \pi k)\}_{0 \leq k<N}$ are distinct at each point $\theta \in[0,2 \pi]$. In particular, this last point of view will be taken here.

For example, a trivial $N$-stranded braid sits in $S^{1} \times \mathbb{C}$ as the graph of $2 \pi N$ periodic map $\gamma: S^{1} \rightarrow \mathbb{C}$ given by

$$
\gamma(\theta)=R^{-1} e^{i \theta / N},
$$

where $R>0$ is any constant.

Reference has been made at the outset to braids in $S^{3}$. Take this to mean the following: View $S^{3}$ as the unit radius sphere about the origin in $\mathbb{C}^{2}$ and identify $S^{1} \times \mathbb{C}$ with its image in $S^{3}$ via the embedding that sends a pair $(\zeta, z)$ to $(\zeta, z) /\left(1+|z|^{2}\right)^{1 / 2} \subset \mathbb{C}^{2}$. This done, an $N$-stranded braid in $S^{3}$ signifies the image of $S^{3}$ of such a braid in $S^{1} \times \mathbb{C}$. 
Two braids in $S^{3}$ are said below to be 'braid isotopic' if they are isotopic through a 1-parameter family of braids.

Step 3: This step constructs an embedded, Lagrangian cylinder in a neighborhood of $S^{1} \times \mathbb{C}$ in $\mathbb{C} \times \mathbb{C}$ that intersects $S^{1} \times \mathbb{C}$ as the given braid $K$. For this purpose, introduce the complex function $\gamma$ on $S^{1}$ that defines $K$ and write $a_{2}=\gamma_{1}+i \gamma_{2}$. With the comments at the end of the preceding step in mind, $K$ can be written as the intersection of $S^{1} \times \mathbb{C} \subset \mathbb{C}^{2}$ with a Lagrangian cylinder defined in a neighborhood in $\mathbb{C}^{2}$ of $S^{1} \times \mathbb{C}$ provided that the following is true: The section $\gamma_{2} d x^{1}+\gamma_{1} d x^{2}$ of $\left.T^{*} \mathbb{C}\right|_{S^{1}}=S^{1} \times \mathbb{C}$ extends to a section of $T^{*} \mathbb{C}$ over a cylindrical neighborhood of the unit circle in the $a_{1}$-plane as the differential of a function that is $2 \pi N$ periodic on the constant radius circles. Thus, the goal is to find a function on the $a_{1^{-}}$ plane, $2 \pi N$ periodic on constant $r$ circles, whose partial derivative in the $x^{1}$-direction restricts to the unit circle as $\gamma_{2}$ and whose partial derivative in the $x^{2}$ direction restricts to the unit circle as $\gamma_{1}$.

To find such a function, it proves useful to introduce the radial coordinates $r \geq 0$ and $\theta$ for the $a_{1}$-plane and write $x^{1}=r \cos \theta$ and $x^{2}=r \sin \theta$. This done, then

$$
\gamma_{2} d x^{1}+\gamma_{1} d x^{2}=\left(\gamma_{2} \cos \theta+\gamma_{1} \sin \theta\right) d r+\left(\gamma_{1} \cos \theta-\gamma_{2} \sin \theta\right) d \theta,
$$

and the task at hand is to find a function, $f$, of $r$ and $\theta$ such that

- $f(r, \theta+2 \pi N)=f(r, \theta)$.

- $\left.\partial_{r} f\right|_{r=1}=\gamma_{2} \cos \theta+\gamma_{1} \sin \theta$.

- $\left.\theta_{\theta} f\right|_{r=1}=\gamma_{1} \cos \theta-\gamma_{2} \sin \theta$.

There is one immediate requirement for $f$ 's existence, which is that

$$
\int_{0}^{2 \pi N}\left(\gamma_{1}(\theta) \cos \theta-\gamma_{2} \sin \theta\right) d \theta=0
$$

since this integral is meant to be $f(1,0)-f(1,2 \pi N)$. In this regard, notice that any given $2 \pi N$ periodic map $\gamma$ to $\mathbb{C}$ with $\{\gamma(\theta+2 \pi k)\}_{0 \leq k<N}$ distinct at all values of $\theta$ can be homotoped through such maps to one that obeys (8). In particular, such a homotopy does not change the braid isotopy class of the corresponding braid. Indeed, if $\gamma: S^{1} \rightarrow \mathbb{C}$ represents an $N$ stranded braid and if $c \in \mathbb{R}$, then $\gamma^{\prime} \equiv \gamma+c e^{-i \theta}$ also has $N$ distinct values at each point for $\left\{\gamma^{\prime}(\theta+2 \pi k)\right\}_{0 \leq k<N}$. Meanwhile, the value of the $\gamma^{\prime}$ version of (8) differs from the value of the $\gamma$ version by $2 \pi N c$ so there is a unique such $\mathrm{c}$ for which the $\gamma^{\prime}$ version of (8) is zero. This understood, agree henceforth to restrict attention to those maps $\gamma$ where (8) holds. 
Given that (8) holds, then there exists a bonafide, $2 \pi N$ periodic function $\sigma$, of $\theta$ whose partial with respect to $\theta$ is equal to $\gamma_{1} \cos \theta-\gamma_{2} \sin \theta$. This understood, then

$$
h \equiv(r-1)\left(\gamma_{2} \cos \theta+\gamma_{1} \sin \theta\right)+\sigma(\theta) .
$$

satisfies the conditions in (7) and so the graph of $d h$ in $T^{*} \mathbb{C}=\mathbb{C}^{2}$ provides an example of the required Lagrangian, at least near the unit circle in the $a_{1}$-plane. As demonstrated in the next step, the Lagrangian defined by (9) is per force embedded near this circle, but perhaps not everywhere.

Step 4: As remarked at the end of the previous step, the Lagrangian cylinder defined by the graph of the differential of the function in (9) may have immersion points where $\left|a_{1}\right|$ differs substantially from 1 . This step and the next describe how to define a properly embedded, Lagrangian cylinder, defined near the $\left|a_{1}\right|=1$ circle and where $\left|a_{1}\right| \geq 1$ that intersects every constant $\left|a_{1}\right|$ slice as a braid that is isotopic to the original.

To start this construction, represent the given braid using, as described, a $2 \pi N$ periodic map $\gamma=\gamma_{1}+i \gamma_{2}: S^{1} \rightarrow \mathbb{C}$ with distinct values for $\{\gamma(\theta+$ $2 \pi k)\}_{0 \leq k<N}$ at all points. By way of shorthand, introduce $\alpha \equiv \gamma_{2} \cos \theta+$ $\gamma_{1} \sin \theta$ and $\beta \equiv 1 \cos \theta-\gamma_{2} \sin \theta$. Note that the pair $(\alpha, \beta)$ are $2 \pi N$ periodic, and the $N$ pairs $\{(\alpha(\theta+2 \pi k), \beta(\theta+2 \pi k))\}_{0 \leq k<N}$ are distinct at each $\theta$ if and only if such is the case for $\{\gamma(\theta+2 \pi k)\}_{0 \leq k<N}$.

Reintroduce the function, $h$, in (9); its partial derivatives determine the Lagrangian cylinder from the preceding step. In particular, these derivatives are

$$
\begin{aligned}
\text { - } \quad \partial_{r} h & =\alpha . \\
\text { - } \partial_{\theta} h & =(r-1) \partial_{\theta}+\beta .
\end{aligned}
$$

By virtue of continuity and the fact that $\{(\alpha(\theta+2 \pi k), \beta(\theta+2 \pi k))\}_{0 \leq k<N}$ has $N$ distinct pairs at each $\theta$, the differential $d h=\partial_{r} h d r+\partial_{\theta} h d \theta$ is such that $\left\{\left.d h\right|_{\theta+2 \pi k}\right\}_{0 \leq k<B}$ also has $N$ distinct values at each point of the constant $r$ circle if $|r-1|$ is not too big. In particular, there exists some $\delta>0$ for which such is the case when $|r-1| \leq 2 \delta$; and this implies that the graph of $d h$ defines an embedded Lagrangian cylinder where $|r-1| \leq 2 \delta$. Of course an upper bound for $\delta$ is determined by the braid map $\gamma$, but there is no positive lower bound to the choice of $\delta$ to use here and in the subsequent discussions. In particular, the condition $\delta<10^{-3}$ is implicitly enforced.

Here is a reformulation of this last point for use below: As long as $|s| \leq$ $2 \delta$, then the graph over $S^{1}$ in $T^{*} \mathbb{C}$ of the 1 -form $\alpha d r+\left(s \partial_{\theta} \alpha+\beta\right) d \theta$ is such 
that its values at the points $\{\theta+2 \pi k\}_{0 \leq k<N}$ are distinct at each $\theta$ and so defines a braid that is braid isotopic to the original (that with $s=0$ ). More generally, as long as $\varepsilon$ is not zero and $|s|<2 \delta$ then the 1-form

$$
\varepsilon \alpha d r+\left(s \partial_{\theta} \alpha+\beta\right) d \theta
$$

also has this same property. Thus, the graph over the circle of the 1-form in (11) defines a braid that is braid isotopic to the original braid defined by $\gamma$.

Step 5: This step uses the observation in (11) to obtain the promised cylinder from Step 4. For this purpose, replace the function in (9) and (10) by

$$
f=\delta(r-1)(r+\delta)^{-1} \alpha+\sigma .
$$

where $\sigma$ is as before, $\partial_{\theta} \sigma=\beta$. This choice gives

$$
\begin{array}{ll}
\text { - } \partial_{r} f=\delta(1+\delta)(r+\delta)^{-2} \alpha . \\
\text { - } \partial_{\theta} f=\delta(r-1)(r+\delta)^{-1} \partial_{\theta} \alpha+\beta .
\end{array}
$$

As before, the graph of df defines a Lagrangian. In particular, with (11) in mind, it follows that $d f$ on any $r \geq 1-2 \delta$ circle has distinct values at $\{\theta+2 \pi k\}_{0 \leq k<N}$ for each $\theta$ and so the graph of $d f$ is a properly embedded, Lagrangian cylinder in the $\left|a_{1}\right| \geq 1-2 \delta$ portion of $\mathbb{C}^{2}$ whose intersection with any constant $\left|a_{1}\right|$ slice is a braid that is braid isotopic to the original.

Step 6: This step explains how to extend the cylinder defined in the previous step to the $\left|a_{1}\right| \leq 1-2 \delta$ portion of $\mathbb{C}^{2}$ capping the $r=1-\delta$ slice of this cylinder with a closed, immersed, Lagrangian disk in the $r \leq 1-\delta$ of $\mathbb{C}^{2}$. The self intersection points of this added disk are described in a subsequent step.

The construction of this extension starts by returning to the example of the trivial $N$ stranded braid where $\gamma(\theta)=e^{i \theta / N}$. This braid has $\gamma_{1}=$ $\cos (\theta / N)$ and $\gamma_{2}=\sin (\theta / N)$ and so (8) is satisfied. Of course, there is a Lagrangian that extends this particular braid, it given by the locus of points $\left(a_{1}, a_{1}^{1 / N}\right)$ in $\mathbb{C}^{2}$ with $a_{1}=r e^{i \theta}$. This extension is given as the graph of $d f_{N}$, where

$$
f_{N}=(1+1 / N)^{-1} r^{1+1 / N} \sin ((1+1 / N) \theta) .
$$

With $f_{N}$ understood, the differential of any function that interpolates between $f$ in (12) where $r \geq 1-\delta$ and $f_{N}$ in (14) where $r$ is near zero defines an immersed Lagrangian disk with the requisite properties. For example,

$$
(1-\chi) f+\chi f_{N}
$$


is such an interpolating function with $\chi$ any function of $r$ that equals 1 near $r=0$ and 1 where $r \geq 3 / 4$.

Step 7: The double points of the Lagrangian defined by (15) can be related directly to properties of the original braid $\gamma$. These relations are described below in Section 2 for a more sophisticated version of the function $f$ that appears in (15). The description of this new $f$ requires the specification of a small and positive constant $\delta$. Given $\delta$, fix a smooth function, $\chi_{\delta}$, of the coordinate $r$ that has the following properties:

- $\chi_{\delta}=1$ where $r \leq 1-2 \delta$.

- $\chi_{\delta}=0$ where $r \geq 1-\delta$.

- $\chi_{\delta}=\delta^{-1}(1-\delta-r)$ where $1-2 \delta+\delta^{2} \leq r \leq 1-\delta-\delta^{2}$.

- $\partial_{r} \chi_{\delta} \leq 0$.

- Set $r^{*} \equiv 1-2 \delta+2 \delta^{4}$ and require that $r^{*}$ be the unique value of $r$ where $\chi_{\delta} r^{1+1 / N}$ achieves its maximum, and require that this maximum be non-degenerate in the sense that

a) $\partial_{r}\left(\chi_{\delta} r^{1+1 / N}\right)>0$ where $r<r^{*}$.

b) $\partial_{r}\left(\chi_{\delta} r^{1+1 / N}\right)<0$ where $r>r^{*}$.

c) $\partial_{r}\left(\chi_{\delta} r^{1+1 / N}\right)=r^{*}-r$ where $r^{*}-\delta^{4}<r<r^{*}+\delta^{4}$.

- Where $1-\delta+\delta^{2}<r \leq 1-\delta$,

a) $\chi_{\delta}\left|\partial_{r} \chi_{\delta}\right|^{-1} \leq 10 \delta^{2}$.

b) $\left|\partial_{r}\left(\chi_{\delta} r^{1+1 / N}\right)\right|$ is decreasing.

- $\partial_{r}^{2} \chi_{\delta}>0$ and $\left|\partial_{r} \chi_{\delta}\right| \leq 100 \delta\left|\partial_{r}^{2} \chi_{\delta}\right|$ where $1-\delta+\delta^{2} / 2 \leq r \leq 1-\delta$.

Note that the third to last point above asks only that $\chi_{\delta} r^{1+1 / N}$ behave in a uniformly quadratic fashion near its maximizer, $r^{*}$. Meanwhile, the final point two points can be achieved by requiring $\chi_{\delta}$ to vanish as $r \rightarrow 1-\delta$ as a multiple of the exponential of the function $-(1-\delta-r)^{-2}$.

Fix a second smooth function, $\chi$, of $\mathrm{r}$ that has value 1 where $r \leq 1 / 2$, value 0 where $r \geq 3 / 4$ and whose derivative is nowhere greater than 8 . 
With the preceding understood, replace the function $f$ in (15), by

$$
f \equiv(1-\chi) f+\chi_{\delta} f_{N} .
$$

By construction, the graph of $d f$ then defines a smooth, properly immersed Lagrangian disk, $L$, in $\mathbb{C}^{2}$ whose $\left|a_{1}\right|>1-\delta$ portion is embedded and intersects every $\left|a_{1}\right| \geq 1-\delta$ slice of $\mathbb{C}^{2}$ transversely as a braid that is braid isotopic to the original one.

In addition, if $\gamma$ is replaced by $\varepsilon \gamma$ with $\varepsilon>0$ and very small, (so representing an isotopy of the original braid to one with distance $\mathcal{O}(\varepsilon)$ from the $a_{1}$-plane), then the graph of the differential of the $\varepsilon \gamma$ version of $f$ in (17) produces a properly immersed, Lagrangian disk in $\mathbb{C}^{2}$ that is embedded near the $\left|a_{1}\right|=1$ slice, embedded where $\left|a_{1}\right| \geq 1$, and intersects every radius 1 or larger 3 -sphere transversely and in a braid that is braid isotopic to the original.

Step 8: Although the Lagrangian defined by the differential of the function in (17) has various virtues, it may not be the most useful for certain applications. This step describes a second Lagrangian in $\mathbb{C}^{2}$ with a somewhat different suite of properties. In particular, the construction here facilitates comparisons when non-isotopic braids differ by a strand crossing. However, the down side here is that the Lagrangians from this step may only intersect all sufficiently large radius spheres as a braid isotopy of the original braid.

To start the construction, choose, as before, a function $\chi$ of the radial coordinate $r$, where now $\chi$ can have value 1 near $r=0$ and value 0 at large $r$. Let $\gamma$ be a given braid and again introduce $\alpha, \beta$ and $\sigma$. With $f_{N}$ as in (14) fix some $\delta>0$ to define

$$
f_{\bullet}(1-\chi)\left(-r^{-1} \alpha+\sigma\right)+\chi \delta f_{N} .
$$

Note that where $r$ is large and so $\chi=0$, the differential of $f_{\bullet}$ is given by

$$
d f_{*}=r^{-2} \alpha d r+\left(-r^{-1} \partial_{\theta} \alpha+\beta\right) d \theta .
$$

The advertised new Lagrangian is defined by the graph of $d f_{*}$. Note that the discussion in Step 4's final paragraph justifies the claim that this new Lagrangian intersects all sufficiently large radius spheres transversely as a braid isotopy of the original braid.

Step 9: This step constructs Lagrangians in $\mathbb{C}^{2}$ that intersect the large radius 3-spheres as a braid isotopy of a given $N$ stranded, but multiple component braid. In particular, after suitably parametrizing the braid, the 
construction is essentially identical to that described in the previous steps. To start, suppose that the braid has some $n$ components, $\left\{\gamma_{1}, \gamma_{2}, \ldots, \gamma_{n}\right\}$ where each $\gamma_{j}$ is a function of $\theta$ that is periodic with period $2 \pi N_{j}$. Here, $\Sigma_{j} N_{j}=N$. Now, take the parameter $\delta$ to be very small, and for each $j$, use the chosen $\delta$ to construct that $\gamma_{j}$ version of the function in either (15), (17). Call it $f_{j}$ and let $L_{j} \subset \mathbb{C}^{2}$ denote the corresponding Lagrangian. Then, the claim is that $L=\cap_{j} L_{j}$ is an immersed Lagrangian with the desired properties.

The proof of this claim requires only a verification that $L$ 's intersection with all large radius 3 -spheres is braid isotopic to the original braid. For this purpose, note that no $\theta \in[0,2 \pi]$ exist where a pair from any $\gamma_{i}$ version of $\{\alpha(\theta+2 \pi k), \beta(\theta+2 \pi k)\}_{0 \leq k<N}$ coincides with one from the $\gamma_{j}$ version when $i \neq j$. This understood, it follows by continuity from (11) that choosing $\delta>0$ and small guarantees that the corresponding $d f_{i}$ and $d f_{j}$ have disjoint graphs where $r \geq 1-\delta$. Thus, $L_{i}$ and $L_{j}$ are disjoint where $r \geq 1$ and their intersection with any $r \geq 1-\delta$ slice of $\mathbb{C}^{2}$ is a braid that is braid isotopic to the original.

An alternate construction takes each $f_{j}$ to be the $\gamma_{j}$ version of the function defined by (19), and then takes $L_{j}$ to be the corresponding immersed, Lagrangian disk. This understood, set $L \equiv \cup_{j} L_{j}$. The latter is immersed, and as follows from (19) using perturbation theory, it intersects all sufficiently large radius 3 -spheres transversely in a braid that is braid isotopic to the original.

Step 10: This step describes how to modify an immersed Lagrangian on some small neighborhood of its immersion points to obtain an embedded, but higher genus Lagrangian. In this regard, note that the resulting Lagrangian may not be orientable. In particular, this situation occurs when the initial Lagrangian has self-intersection points with positive local degree. By the way, the existence of such a modification has surely been known for years by experts, but as the construction is relatively straightforward, it is worth relating the details.

The first point to make is that any immersed Lagrangian can be modified on any given neighborhood of its singular points (with out changing the genus) so that the result has only transversal, double point self-intersections. Moreover, if the original singular set is compact, then this modification produces only a finite set of such intersections. The definition of such a modification exploits the 4-dimensional version of the following basic and well known lemma:

Lemma: 1 . $i 1_{\dot{\delta}}$ Let $n \geq 1$ be an integer, $X$ be a $2 n$-dimensional mani- 
fold with a symplectic form, and $L \subset X$ be an $n$-dimensional Lagrangian submanifold. Then each point of $L$ has a neighborhood with coordinates $\left(x^{1}, \ldots, x^{n}, p_{1}, \ldots, p_{n}\right)$ in which the $\left\{p_{j}=0\right\}_{1 \leq j \leq n}$ slice is $L$ and to which the symplectic form restricts as $\Sigma_{j} d p_{j} d x^{j}$.

Given the lemma, a first perturbation of $L$ puts a neighborhood of some given singular point in the appropriate form. For this purpose, select a singular point $z \in L$ and take a coordinate system centered on this point as described by the lemma. A perturbation of $L$ near $z$ is defined by the locus where $\left\{p_{j}=\partial_{j} h\right\}$ where $h$ is a function on $L$ whose partial derivatives are small. In particular, as there are functions defined near the origin in $\mathbb{R}^{n}$ with any given vector as differential at 0 and any given symmetric matrix as Hessian, Sard's theorem provides perturbations of $L$ that stay arbitrarily close to $L$, contains $z$ and result in a new Lagrangian, $L^{\prime}$, with a transversal and purely double point self-intersection at $z$.

This local construction understood, a straightforward extension produces a Lagrangian with all singular points as desired. The details of the extensions are tedious and omitted.

Given that a singular point in the Lagrangian $L$ is isolated and a transversal double point, the modification to make a Lagrangian that is embedded with one less singular point procedes as follows: First, fix one of the sheets of the Lagrangian on a neighborhood of a singular point and introduce the Lemma's coordinates with center on the singular point. This done, the other sheet can be perturbed without introducing new singular points so that it intersects the original in the origin of these coordinates and so that a neighborhood of the origin in this sheet coincides with the locus where $x^{1}=x^{2}=0$. This understood, introduce the complex coordinates $a_{1}=x^{1}+i x^{2}$ and $a_{2}=p_{2}+i p_{1}$ with respect to which the symplectic form is given by (3) and $L$ 's intersection with a neighborhood of the origin is the locus where $a_{1} a_{2}=0$.

Now, consider the perturbation of $L$ in this neighborhood given by the locus where $a_{1} a_{2}=i \varepsilon$ with $\varepsilon$ some non-zero, small positive constant. The latter locus is a smooth, Lagrangian submanifold. Moreover, if $\varepsilon$ is small, then its intersection with the complement of a small radius ball about the origin consists of two annuli, one very close to the $a_{1}$-plane and the other close to the $a_{2}$-plane. Here, the annulus that near the $a_{1}$-plane is the locus of points where $p_{1}=\varepsilon \partial_{1} \ln (r)$ and $p_{2}=\varepsilon \partial_{2} \ln (r)$ where $r=\left(x_{1}^{2}+x_{2}^{2}\right)^{1 / 2}$. Meanwhile, the annulus that is close to the $a_{2}$-plane is defined by the analogous locus where the role of the pair $\left(x^{1}, x^{2}\right)$ is switched with that of $\left(p_{1}, p_{2}\right)$. 
These last points understood, fix a non-increasing function $\chi$ on $[0, \infty)$ that equals 1 on $[0,1]$, vanishes on $[3, \infty)$ and whose derivative is no larger in absolute value than 1 . This done, replace the annulus close to the $a_{1}$-plane by the locus of points where $p_{1}$ and $p_{2}$ are the respective partial derivatives of $\varepsilon \chi(r / \varepsilon) \ln (r)$. At the same time, replace the annulus close to the $a_{2}$-plane in the analogous fashion. The result is, for small $\varepsilon$, a Lagrangian that has one less self-intersection point than the original and agrees with the original in the complement of a small neighborhood of the chosen self-intersection point.

By the way, this construction respects the given orientation on the two intersecting sheets of the original Lagrangian only when the local intersection number of the two sheets is +1 . For topological reasons, it is impossible to remove a local intersection with intersection number -1 using a local modification that preserves the orientations on the intersecting sheets.

\section{Immersion double points and crossings}

The self intersection points of the Lagrangians defined from either (15), (17) or (19) can be directly related to properties of the original braid. This is done here for the small $\delta$ versions of the Lagrangian given by (17).

To start, suppose that the constant $\delta$ that appears in (12) and (17) is taken very small (remember that the discussion in Section 1 is valid as long as $\delta$ is positive no matter how small). The fact is that the integer $N$ determines an upper bound for the application that follows, but such an upper bound is not explicitly derived. With $\delta$ small, replace the map $\gamma$ that defines a given braid by $\varepsilon \gamma$ with $\varepsilon>0$ and very small. This done, then the self-intersection points of the Lagrangian from the resulting $f$ in (17) can be interpreted in terms of the crossings of the original braid. The purpose of this section is to explain how this comes about. The discussion that follows is divided into six parts.

Part 1: To begin the story, remark that with $\gamma$ given and the function $f$ defined from $\gamma$ as in (12) then

$$
f_{\varepsilon} \equiv(1-\chi) \varepsilon f+\chi_{\delta} f N .
$$

is the $\varepsilon \gamma$ version of (17). Now, note that $f_{\varepsilon}=f_{N}$ where $r \leq 1 / 2$ and so the Lagrangian that is defined by $d f_{\varepsilon}$ intersects the $\left|a_{1}\right|<1 / 2$ portion of $\mathbb{C}^{2}$ as an embedded disk. When $\varepsilon$ is small, such is the case where $\left|a_{1}\right|<1-2 \delta$ for the following reason: Where $r \leq(1-2 \delta)$, the differential of $f_{\varepsilon}$ differs from that 
of $\varepsilon, f_{N}$ by a term no larger than $\varepsilon|d f|$, and so for small the $\left|a_{1}\right| \leq(1-2 \delta)$ portion of the Lagrangian defined by $d f_{\varepsilon}$ is a small perturbation of that defined by $d f_{N}$. In particular, as the latter is embedded, so the $\left|a_{1}\right|<1-2 \delta$ portion of the former is also.

With the preceding understood, it follows that the immersion points of the Lagrangian in question all lie where $1-2 \delta \leq\left|a_{1}\right| \leq 1$. To study these points, note first that the function $f_{\varepsilon}$ where $1-2 \delta \leq r \leq 1$ is given by $f_{\varepsilon}=\varepsilon f+\chi_{\delta} f_{N}$, and so self-intersection points in the graph of $d f_{\varepsilon}$ are at $(r, \theta)$ where the values at $\{(r, \theta+2 \pi k)\}_{0 \leq k<N}$ of the $2 \pi N$ periodic 1 -form

$$
\left[\varepsilon \partial_{r} f+\mu^{-1} \partial_{r}\left(\chi_{\delta} r^{\mu}\right) \sin (\mu \theta)\right] d r+\left[\varepsilon \partial_{\theta} f+\chi_{\delta} r^{\mu} \cos (\mu \theta)\right] d \theta
$$

are not pairwise distinct. To investigate where these $(r, \theta)$ occur, it proves useful to separate the search into three regimes. The first occurs where $1-2 \delta+\delta^{2} \leq r \leq 1-\delta-\delta^{2}$, the second where $1-2 \delta<r<1-2 \delta+\delta^{2}$ and the third where $1-\delta-\delta^{2}<r<1-\delta$.

Part 2: In the first regime, (16) implies that the 1-form in (21) looks like

$$
\delta^{-1}\left[\mu^{-1} \sin (\mu \theta)+\mathcal{O}(\varepsilon+\delta)\right] d r+\left[\delta^{-1}(1-2 \delta+r) \cos (\mu \theta)+\mathcal{O}(\varepsilon)\right] d \theta
$$

In particular, as the values at $\{\theta+2 \pi k\}_{0 \leq k<N}$ of the pair $(\cos (\mu \theta), \sin (\mu \theta))$ define a set of $N$ distinct elements for each $\theta$, so do the values of the form in (19) at $\{(r, \theta+2 \pi k)\}_{0 \leq k<N}$ when both $\delta$ and $\varepsilon$ are small.

Part 3: Consider next the second regime, that where $1-2 \delta \leq r \leq 1-2 \delta+\delta^{2}$. Here, $\chi_{\delta}$ is close to 1 but the deriviative of $\chi_{\delta} r^{\mu}$ has a zero so the 1 -form in (21) appears schematically as

$$
\left[\varepsilon \delta_{r} f+\mu^{-1} \partial_{r}\left(\chi_{\delta} r^{\mu} \sin (\mu \theta)\right] d r+[\cos (\mu \theta)+\mathcal{O}(\varepsilon)] d \theta .\right.
$$

Now, given (23) and small $\varepsilon$, perturbation theory precludes less than $N$ distinct elements in the set of values of (23) at a given $\{(r, \theta+2 \pi k)\}_{0 \leq k<N}$ unless $\theta$ is close to a point where $\{\cos (\mu \theta+2 \pi k / N)\}_{0 \leq k<N}$ has less than $N$ distinct elements. In this regard, a glance at the graph of the cosine function indicates that there are $2(N-1)$ points in $[0,2 \pi]$ where $\{\cos (\mu \theta+2 \pi k)\}_{0 \leq k N N}$ has less than $N$ distinct elements and at such points, this set has precisely $N-1$ distinct elements. Moreover, the coincidence of a pair of elements of this set at these special $\theta$ points is achieved in a manner that is nondegenerate in the following sense: If $\theta_{*} \in[0,2 \pi]$ is one of these special points, and if $k_{*} \in\{1, \ldots, 2 \pi N\}$ is such that $\cos \left(\mu \theta_{*}+2 \pi k_{*} / N\right)=\cos \left(\mu \theta_{*}\right)$, then the derivative of $\cos \left(\mu \theta+2 \pi k_{*} / N\right)-\cos (\mu \theta)$ at $\theta_{*}$ is non-zero. 
By the way, this count of points in $[0,2 \pi]$ where $\{\cos (\mu \theta+2 \pi k / N)\}_{0 \leq k<N}$ has less than $N$ distinct elements arises from the fact that each $k \in\{1, \ldots, N-$ $1\}$ determines precisely two values for $\theta \in[0,2 \pi]$ where $\cos (\mu \theta)$ and $\cos (\mu \theta+$ $2 \pi k / N)$ are equal.

These last remarks understood, an application of perturbation theory finds, for small $\varepsilon$, precisely $2(N-1)$ points $\theta \in[0,2 \pi]$ for which the set of values of the $d \theta$ component in (20) at $\{(\theta+2 \pi k)\}_{0 \leq k<N}$ has less than $N$ distinct elements, and at such a point, this set then has precisely $N-1$ elements. Moreover, each such point in $[0,2 \pi]$ will be very close (for small $\varepsilon)$ to a point where there are fewer than $N$ distinct elements in the set of values of $\cos (\mu \theta)$ at $\{\theta+2 \pi k\}_{0 \leq k<N}$. Use $\Lambda \subset[0,2 \pi]$ denote the set of those $\theta$ where the $d \theta$ component of $(20)$ at $\{\theta+2 \pi k\}_{0 \leq k<N}$ has fewer than $N$ elements.

As demonstrated by a second application of perturbation theory, the fifth point of (16) has the following implication: Given that $\delta$ is small and then $\varepsilon$ is very small, each $\theta_{*} \in \Lambda$ is the $\theta$-component of a unique point $\left(r_{*}, \theta_{*}\right)$ with $r_{*} \in\left[r^{*}-\delta^{4}, r^{*}+\delta^{4}\right]$ where the set of values of the whole 1-form in (23) at $\left\{\left(r_{*}, \theta_{*}+2 \pi k\right)\right\}_{0 \leq k<N}$ has less than $N$ (and thus $N-1$ ) elements.

Given all of the above, then it follows that the Lagragian in $\mathbb{C}^{2}$ defined by the differential of the small $\delta$ and very small $\varepsilon$ version of $f_{\varepsilon}$ in $(20)$ has precisely $2(N-1)$ double points where $\left|a_{1}\right|$ lies between $1-\delta+\delta^{2}$ and $1-2 \delta$. Moreover, the arguments ust given establish that each of these self intersection points of the Lagrangian is transversal. Meanwhile, the discussion below in Part 6 explains why these self intersection points all contribute the same local sign to any count of a self intersection number of $L$.

Part 4: Consider now the third regime, that where $1-\delta-\delta^{2} \leq r<1-\delta$. Here, it proves useful to break this regime into two parts, the first where $\chi_{\delta} \geq \delta \varepsilon$ and the second where this last condition does not hold. In this first regime, $\partial_{r} \chi_{\delta}=-10^{-1} \delta^{-1} \varepsilon w$ where the function $\mathrm{w}$ is greater than 1 by virtue of the second to last point in (16). Thus, (21) has the schematic form

$$
10^{-1} \delta^{-1} \varepsilon\left[-w \mu^{-1} \sin (\mu \theta)+\mathcal{O}(\delta)\right] d r+\left[\varepsilon \partial_{\theta} f+\chi_{\delta} r^{\mu} \cos (\mu \theta)\right] d \theta .
$$

In particular, when $\delta$ is small, then the values of the $d r$ component of (24) at the points in $\{(r, \theta+2 \pi k)\}_{0 \leq k<N}$ is a set of fewer than $N$ distinct elements provided that $\theta$ is near one of the $2(N-1)$ points where $\left\{(\sin (\mu \theta+2 \pi k)\}_{0 \leq k N N}\right.$ has fewer than $N$ distinct elements.

Now, not all of these $2(N-1)$ points in $[0,2 \pi]$ correspond to selfintersection points of the Lagrangian with $r$ in the prescribed range. Indeed, 
when $\varepsilon$ and $\delta$ are very small, then the form of the $d \theta$ component of (24) forces a self-intersection point at $(r, \theta)$ with $\theta$ very close to such a $\theta_{*}$ and with $r$ in the prescribed range provided that the following requirment is met: The expressions

$$
\beta\left(\theta_{*}+2 \pi k\right)-\beta\left(\theta_{*}+2 \pi k^{\prime}\right) \text { and } \cos \left(\mu \theta_{*}+2 \pi k / N\right)-\cos \left(\mu \theta_{*}+2 \pi k^{\prime} / N\right)
$$

have opposite sign when $k \neq k^{\prime}\{0, \ldots, N-1\}$ are chosen to make $\sin \left(\mu \theta_{*}+\right.$ $2 \pi k)=\sin \left(\mu \theta_{*}+2 \pi k^{\prime}\right)$. Moreover, if the left most difference in (25) is nonzero for all of the $2(N-1)$ possibilities for $\theta_{*}$, then perturbation theory guarantees a $1-1$ correspondence between the self intersection points in the third regime and those $\theta_{*}$ where the just stated requirement is met. This guarantee also comes with a rider to insure that these self intersection points are all transverse double points.

Part 5: Consider the final part of the third regime where $\chi_{\delta}<\delta \varepsilon$. Here, (21) has the schematic form

$$
\left[\varepsilon \delta(1+\delta)(r+\delta)^{-2} \alpha+\mu^{-1} \partial_{r}\left(\chi_{\delta} r^{\mu} \sin (\mu \theta)\right] d r+\varepsilon[\beta+\mathcal{O}(\delta)] d \theta .\right.
$$

In this regard, note that when $\delta$ is small, then the $d \theta$ component of (26) has $N$ distinct values except possibly near points in $[0,2 \pi]$ where the function $\beta$ has fewer than $N$ distinct values.

Introduce the term 'twisted crossing point' to denote a point $\theta_{*} \in[0,2 \pi]$ where the set $\left\{\beta\left(\theta_{*}+2 \pi k\right)\right\}_{0 \leq k<N}$ has less than $N$ distinct values. A twisted crossing point $\theta_{*}$ is transverse when two requirments are met. The first is met when $\left\{\beta\left(\theta_{*}+2 \pi k\right)\right\}_{0 \leq k<N}$ has precisely $N-1$ distinct elements. Assuming now that the first requirement is met, let $k \neq k^{\prime}$ denote the two integers in $\{0, \ldots, N-1\}$ for which the value of $\beta$ at $\theta_{*}+2 \pi k$ is the same as that at $\theta_{*}+2 \pi k^{\prime}$ agree. The second requirement is then met when the difference the locally defined function $\beta(\theta+2 \pi k)-\beta\left(\theta+2 \pi k^{\prime}\right)$ has non-zero derivative at $\theta=\theta_{*}$.

If the braid is such that its twisted crossing points are all transverse, then those points where the $d \theta$ component of $(26)$ has less than $\mathrm{N}$ distinct values are in $1-1$ correspondence with the set of twisted crossing points. Indeed, with this transversality assumption, the final point in (16) guarantees that each point of the one set is very close to precisely one point in the other.

Now, given the preceding comments, the final point in (16) has the following implication: Let $\theta_{*}$ be a twisted crossing point and let $k \neq k^{\prime} \in$ $\{0, \ldots, N-1\}$ denote the unique pair for which $\beta\left(\theta_{*}+2 \pi k\right)=\beta\left(\theta_{*}+2 \pi k^{\prime}\right)$. If

$$
\alpha\left(\theta_{*}+2 \pi k\right)-\alpha\left(\theta_{*}+2 \pi k^{\prime}\right) \text { and } \sin \left(\mu \theta_{*}+2 \pi k / N\right)-\sin \left(\mu \theta_{*}+2 \pi k^{\prime} / N\right)
$$


have the same sign, then such a $\theta_{*}$ corresponds to a unique $\theta \in[0,2 \pi]$ near $\theta_{*}$ and a unique $r \in\left[1-\delta-\delta^{2}, 1-\delta\right]$ where (27) has less than $N$ distinct points at $(r, \theta)$. Furthermore, this then defines a $1-1$ correspondence between twisted crossing points that obey (27) and points $(r, \theta)$ with $1-\delta-\delta^{2} \leq r<1-\delta$ where (27) has less than $N$ distinct values.

Part 6: This final part of the story explains how to use information from the braid to compute the local sign of at the various self intersection points of the Lagrangian. For this purpose, agree to orient the Lagrangian as a (multi-valued) graph over the $a_{1}$-plane, where the latter is oriented by the form $r d r d \theta$. This is to say that the Lagrangian is to be viewed as the graph of the differential of $f_{\varepsilon}$ in $(20)$.

Now, suppose a transverse double point occurs in $L$ over a point in the $a_{1}$-plane with coordinates $\left(r_{*}, \theta_{*}\right)$. Thus, two sheets of $L$ intersect at this point and so there exists a distinct pair $k, k^{\prime} \in\{0, \ldots, N-1\}$ such that $d f_{\varepsilon}$ has the same value at $\left(r_{*}, \theta_{*}+2 \pi k\right)$ and at $\left(r_{*}, \theta_{*}+2 \pi k^{\prime}\right)$. This understood, it then follows that the sign $( \pm 1)$ of this self intersection is equal to minus the sign of the determinant of the hessian at $\left(r_{*}, \theta_{*}\right)$ of the function

$$
H \equiv f_{\varepsilon}(r, \theta+2 \pi k) f_{\varepsilon}\left(r, \theta+2 \pi k^{\prime}\right) .
$$

Apply this prescription to the 2(N-1) self intersection points described above in Part 3 to find that each has local intersection number -1 . Indeed, to order $\mathcal{O}(\varepsilon)$, the function $H$ is the same as $H_{0} \equiv \chi_{\delta} r^{\mu}\left(\sin \left(\mu \theta_{*}+2 \pi k\right)-\right.$ $\left.\sin \left(\mu \theta_{*}+2 \pi k^{\prime}\right)\right)$, and so small $\varepsilon$ makes both the differential and hessian of $H$ very close to those of $H_{0}$. Thus, small $\varepsilon$ makes each of the relevant critical points of $H$ very close to one of $H_{0}$ and it makes the signs of the corresponding determinants agree if $H_{0}$ 's determinant is not zero. In this regard, note that $\mathrm{H} 0$ has positive determinant at each relevant critical point because each occurs where $\chi_{\delta} r^{\mu}$ is maximized.

Consider next the signs of the self intersection points that are described above in Part 4. In this regard, it follows from (24) that when $\varepsilon$ and $\delta$ are very small, then the sign of the relevant determinant is negative. Indeed, this follows because the hessian in question differs by $\mathcal{O}(\varepsilon \delta)$ from a matrix having the form $\varepsilon \mathcal{H}$, where $\mathcal{H}$ is the symmetric matrix with zeros on the diagonal and, in the notation from Part 4 , with off diagonal entries equal to $-w\left(\cos \left(\mu \theta_{*}+2 \pi k\right)-\cos \left(\mu \theta_{*}+2 \pi k^{\prime}\right)\right)$. Thus, all of Part 4's self- intersection points have local intersection sign equal to +1 .

Turn at last to the self-intersection points that are described above in Part 5. Under the assumptions that all of the twisted crossing points $\left\{\theta_{*}\right\}$ are non-degenerate and that $\varepsilon$ and $\delta$ are both small, then the local intersection 
signs are determined as follows: Suppose that a twisted crossing point $\theta_{*}$ determines a self intersection point as described in Part 5 . Then, the local intersection number for this intersection point is minus the product of the sign of $\alpha\left(\theta_{*}+2 \pi k\right)-\alpha\left(\theta_{*}+2 \pi k^{\prime}\right)$ with the sign of the derivative at the point $\theta=\theta_{*}$ of $\beta(\theta+2 \pi k)-\beta\left(\theta+2 \pi k^{\prime}\right)$. Here, $k$ and $k^{\prime}$ are as given in (27). (The latter all follows with the help of the final point in (16).)

By the way, this sign can be interpreted as follows: View the triple $(\alpha, \beta, \theta)$ as the coordinates of a portion of the braid in $\mathbb{R}^{3}$, and then view $(\beta, \theta)$ as the coordinates of the braid's projection into $\mathbb{R}^{2} \subset \mathbb{C}$. This done, then a twisted crossing point corresponds to a crossing of strands as viewed via the direction defined by this projection. Now, orient the the strand using the 1-form $d \theta$. This understood, the strand that corresponds near $\theta=\theta_{*}$ to the parameterization by $\theta \rightarrow(\alpha(\theta+2 \pi k), \beta(\theta+2 \pi k))$ passes on top of the other strand with respect to this projection when $\alpha\left(\theta_{*}+2 \pi k\right)-\alpha\left(\theta_{*}+2 \pi k^{\prime}\right)>$ 0 and passes under the other strand when $\alpha\left(\theta_{*}+2 \pi k\right)-\alpha\left(\theta_{*}+2 \pi k^{\prime}\right)<0$. This understood, the sign of the corresponding self intersection point is positive when the crossing as seen by this projection appears as in the following diagram:

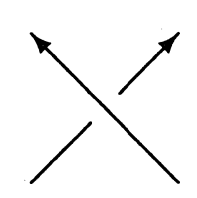

\section{Lagrangians and crossing changes}

Suppose that two braids differ by a single strand crossing. As certain knot invariants can be characterized in terms of skein relations, one might ask how the corresponding Lagrangians compare with each other, and with that for the third braid in the skein diagram. To be more precise, suppose that the three braids are identical except for their intersection with a fixed small ball in $S^{1} \times \mathbb{C}$, and in this ball, the three braids correspond to the following 
three pictures:

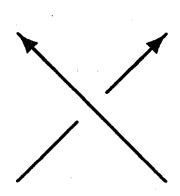

$\gamma_{+}$

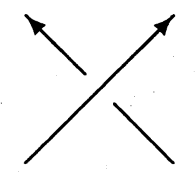

$\gamma_{-}$

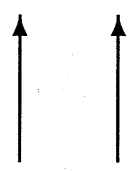

$\gamma_{0}$

This question is considered below when the Lagrangians for the braids $\gamma_{ \pm}$are such that they are given at large values of $r=\left|a_{1}\right|$ as the graph of the differential of the appropriate version of the function $f_{*}$ depicted in (19).

Some conventions need setting to connect the pictures in (30) with $f_{*}$. For this purpose, suppose that when $\gamma$ is one the braid in one of the pictures in (30), then the pair $(\alpha, \theta)$ give the $x$ and $y$ coordinates of the strands in (30). In this regard, the convention is standard: The variable $x$ increases with horizontal motion to the right in (30) and $y$ increases with vertical motion to the top of the drawing in (30). This understood, then $\beta$ should be assumed to increase in the direction out of the paper but away from the reader. (Note that the drawing in (29) uses the different convention where $(\beta, \theta)$ are the coordinates of the projection.)

Now, to simplify notation, suppose that $\theta_{*}=0$ is the value of the $\theta$ coordinate where the projection in the $\gamma_{+}$diagram in (30) has one strand pass over the other. In this regard, note that $\gamma_{+}$can be isotoped as a braid so that its parametrization at values of $\theta$ near 0 is such that the under passing strand in the $\gamma_{+}$diagram is described by $\left(\alpha_{+}(\theta)=\theta, \beta_{+}(\theta) \tau(\theta)\right)$ where $\tau$ is a smooth, non-negative function of $\theta$ that is positive at $\theta=0$ and vanishes where $\theta$ is near the top and bottom of its implicit range in (30). To be precise, suppose that this range for $\theta$ is $(-\varepsilon, \varepsilon)$ and that $\tau(\theta)=0$ for $|\theta|>\varepsilon / 2$. Meanwhile, the over passing strand in the $\gamma_{+}$picture is described by $\left(\alpha_{+}(\theta+2 \pi k)=-\theta, \beta_{+}(\theta+2 \pi k) \equiv 0\right)$ where $k \in\{1, \ldots, N-1\}$.

At the same time, $\gamma_{-}$can be isotoped as a braid so that for $\theta$ near 0 , the parametrizing data $\left(\alpha_{-}, \beta_{-}\right)$has $\left(\alpha_{-}(\theta), \beta_{-}(\theta)\right)=(\theta,-\tau(\theta))$ and $\left(\alpha_{-}(\theta+\right.$ $\left.2 \pi k), \beta_{-}(\theta+2 \pi k)\right)$ equal to $(-\theta, 0)$. Thus, values of $\theta$ near zero in both the $\gamma_{+}$and $\gamma_{-}$diagram parametrize the strand that points up and to the right.

Having digested this notation, define the family $\left\{\gamma_{s}: s \in[-1,1]\right\}$ of maps from $S^{1}$ to $\mathbb{C}$ as follows: When $\theta \notin(-\varepsilon, \varepsilon)$, then $\gamma_{s}(\theta)=\gamma_{+}(\theta)=\gamma_{-}(\theta)$. On 
the other hand, when $\theta \in(-\varepsilon, \varepsilon)$, then

$$
\left(\alpha_{s}(\theta)=\theta, s \tau(\theta)\right) .
$$

This understood, then all positive $\mathrm{s}$ versions of $\gamma_{s}$ define a braid that is isotopic to $\gamma_{+}$while all negative s versions define one that is isotopic to $\gamma_{-}$.

Now consider the $\gamma_{s}$ version, $L_{s}$, of the Lagrangian defined in Step 8 of Section 1 via the differential of (18)'s function $f_{*}$. In particular, the small, but positive $\mathrm{s}$ versions have a transversal double point that is parametrized by $\theta=0$ and $r=2 \tau(0) / s$ and otherwise, no double points where $r \geq R_{0}$ with $R_{0}$ independent of $s$. Meanwhile, the $s<0$ versions of $L_{s}$ have no double points at all where $r \geq R_{0}$.

Note next that even for $s=0$, the definition given in Step 8 of Section 1 for $L_{s}$ makes perfectly good sense and describes a properly immersed, Lagrangian disk in $\mathbb{C}^{2}$. In particular, $L_{s=0}$ can be assumed to have solely transversal and isolated double points if the braid $\gamma_{+}$is chosen in a suitably generic fashion in the complement of the ball pictured in (30). In any event, the non-zero versions of $L_{s}$ can be assumed to converge in the $C^{\infty}$ topology as $s \rightarrow 0$ to the $s=0$ version.

In fact, these last conclusions about $L_{s}$ can be strengthened as follows: The Lagrangian $L_{0}$ can be assumed to have the same double points as all small and negative $s$ versions of $L_{-s}$ and be isotopic by small Hamiltonian isotopies of $\mathbb{C}^{2}$ to such $L_{s}$. It can also be assumed to intersect all spheres with radius greater than $R_{0}$ transversely in a braid that is braid isotopic to $\gamma_{-}$. Meanwhile, Ls for small and positive $\mathrm{s}$ can be assumed to intersect all spheres with radii between $R_{0}$ and $2 \tau(0) / s$ transversely, and in a braid that is also braid isotopic to $\gamma_{-}$even as it intersects all spheres of radius greater than $2 \tau(0) / s$ in a braid that is isotopic to $\gamma_{+}$. Moreover, the portion of such a small and positive s version of $L_{s}$ where $r$ is less than $2 \tau(0) / s$ can be taken to be isotopic via proper, Hamiltonian isotopy of $\mathbb{C}^{2}$ to the same portions of the small, but negative s versions of $L_{s}$.

This said about the $\gamma_{+}$and $\gamma_{-}$Lagrangians, what follows is a description of a related Lagrangian, $L$, for the braid $\gamma_{0}$ in (30). For this purpose, fix some $r_{0} \gg R_{0}$. Then $L$ has the following properties:

- The portion of $L$ where $r<r_{0}$ is isotopic via a Hamiltonian isotopy to $L_{0}$, while the portion where $r>r_{0}$ is likewise isotopic to the $\gamma_{0}$ version of the Lagrangian from $f_{*}$. 
- In fact, given some positive $\varepsilon>0$, the $r<r_{0}-\varepsilon$ portion of $L$ can be taken equal to $L_{0}$, while the $r>r_{0}+\varepsilon$ portion can be taken equal to the same portion of the $\gamma_{0}$ version of the Lagrangian from Step 8 in Section 1.

- $L$ is embedded where $r \geq R_{0}$, but this portion of $L$ is not a cylinder, and thus not a multi-valued graph over the $a_{1}$-plane. Rather, the $r \geq R_{0}$ portion of $L$ projects to the $a_{1}$ plane with a single ramification point to account for the change in the topology of its constant $r$ slices at $r=r_{0}$.

The story on $L$ starts with a digression to provide a local model for this ramification business. For this purpose, consider the locus in $\mathbb{C}^{2}$ where

$$
a_{1}-r_{0}=a_{2}^{2} \text {. }
$$

Note that this locus defines a smooth, Lagrangian surface in $\mathbb{C}^{2}$ whose projection to the $a_{1}$-plane is $2-1$ save for the single critical point that projects to $\left(r=r_{0}, \theta=0\right)$.

To see how (33) models the desired behavior $\theta$ near zero, view the pair $\alpha$ and $\beta$ with $\theta$ as coordinates on $S^{1} \times \mathbb{C}^{2}$. Then, where $r$ is near $r_{0}$ and $\theta$ near 0 , the equation in (33) has the schematic form

- $r-r_{0}=\alpha^{2}-\beta^{2}+\cdots$,

- $\theta=2 r_{0}^{-1} \alpha \beta+\cdots$,

where the '...' signify terms that are $\mathcal{O}\left(\alpha^{4}+\beta^{4}\right)$.

This last equation understood, first fix $r$ at some value very near, but less than $r_{0}$ and then view the resulting locus as a curve in the $(\alpha, \theta, \beta)$ version of $\mathbb{R}^{3}$. In particular, note that view from the same vantage as that in (30) looks like the $\gamma_{-}$picture in (30). Meanwhile, the analogous view for the locus defined by fixing $r$ near, but greater than $r_{0}$ in (34) looks like the $\gamma_{0}$ version of $(30)$.

Given the comments in the preceding paragraph, the task to construct $L$ as described in (32) is straightforward and left to the reader with the hints to take $\alpha$ and $\beta$ to be very small near $\theta=0$ when comparing with the description of the Lagrangian $L_{0}$. 


\section{Lagrangians in $O(-1) \oplus O(-1)$}

As in the introduction, let $O(-1) \rightarrow \mathbb{P}^{1}$ denote the degree -1 , holomorphic line bundle over the Riemann sphere. The purpose of this section is to describe how certain Lagrangians from the previous section can be used to construct a 3-dimensional Lagrangian in the Kähler manifold $O(-1) \oplus O(-1)$. The construction starts with a 2-dimensional Lagrangian, $L \subset \mathbb{C}^{2}$, that is mapped to itself under multiplication by -1 on $\mathbb{C}^{2}$ and produces a 3 dimensional Lagrangian in $O(-1) \oplus O(-1)$ that projects to the equator in $\mathbb{P}^{1}$ with fiber $L$.

The symplectic form for the space $O(-1) \oplus O(-1)$ is a standard Kähler form. To view it, introduce the homogeneous complex coordinates $(z, w) \in$ $\mathbb{C}^{2}-\{0\}$ for $\mathbb{P}^{1}$. Thus, $(z, w)$ gives the same point in $\mathbb{P}^{1}$ as $(\lambda z, \lambda w)$ when $\lambda \in$ $\mathbb{C}$ is not zero. Now introduce the homogeneous coordinates $\left((z, w), \eta_{1}, \eta_{2}\right)$ for $O(-1) \oplus O(-1)$ where now the latter and $\left((\lambda z, \lambda w), \lambda^{-1} \eta_{1}, \lambda^{-1} \eta_{2}\right)$ give the same point. This done, introduce the coordinates $\zeta_{i}=\left(|z|^{2}+|w|^{2}\right)^{1 / 2} \eta_{i}$; the latter transform as $\zeta_{i} \rightarrow|\lambda| \lambda^{-1} \zeta_{i}$ when $(z, w) \rightarrow(\lambda z, \lambda w)$. In particular, the transformation for each $\zeta_{i}$ is unitary, so the norm $\left|\zeta_{i}\right|$ gives a well defined function.

Next, introduce

$$
\pi_{i} \equiv d \zeta_{i}+A \zeta_{i}
$$

where $A$ is the connection 1 -form

$$
A=\left(|z|^{2}+|w|^{2}\right)^{-1} \operatorname{Im}(\bar{z} d z+\bar{w} d w)
$$

thus $\pi_{i}$ transforms as does $\zeta_{i}$ when $(z, w) \rightarrow(\lambda z, \lambda w)$ with $\lambda$ now any nowhere zero, complex valued function. Letting $u \rightarrow(z=u, w=1)$ denote the complex coordinate on the $w \neq 0$ portion of $\mathbb{P}^{1}$, then $A=$ $\left(|u|^{2}+1\right)^{-1} \operatorname{Im}(\bar{u} d u)$ and $d A=\left(|u|^{2}+1\right)^{-2} \operatorname{Im}(d \bar{u} \wedge d u)$.

With the $\left\{\pi_{i}\right\}$ in hand, the symplectic form on $O(-1) \oplus O(-1)$ is written using the coordinate $u$ as

$$
i 2^{-1}\left(\pi_{1} \wedge \bar{\pi}_{1}+\pi_{2} \wedge \bar{\pi}_{2}+\left(|u|^{2}+1\right)^{-2}\left(a+\left|\zeta_{1}\right|^{2}+\left|\zeta_{2}\right|^{2}\right) d u \wedge d \bar{u}\right) ;
$$

here $a>0$ and $2 \pi a$ gives the symplectic area of $\mathbb{P}^{1}$. For reference in the subsequent discussion, note that this symplectic form restricts to the $|u|=1$ equator in $\mathbb{P}^{1}$ as

$$
i 2^{-1} \Sigma_{i} d\left(e^{i \varphi / 2} \zeta_{i}\right) \wedge d\left(e^{-i \varphi / 2} \bar{\zeta}_{i}\right)
$$


where $\varphi \in[0,2 \pi]$ is the argument of $u$.

Now let $L \subset \mathbb{C}^{2}$ denote an immersed, Lagrangian surface that is mapped to itself by the action of multiplication by -1 on $\mathbb{C}^{2}$. With (38) understood, it follows directly that

$$
M \equiv\left\{\left(u=e^{i \varphi}, \zeta_{1}, \zeta_{2}\right):\left(e^{i \varphi / 2} \zeta_{1}, e^{i \varphi / 2} \zeta_{2}\right) \in L\right\}
$$

is an immersed Lagrangian in $O(-1) \oplus O(-1)$. As an abstract manifold, $M$ is diffeomorphic to the quotient of $S^{1} \times L$ by the action of $\mathbb{Z} / 2$ that sends $\left(\varphi, z_{1}, z_{2}\right)$ to the point $\left(\varphi+\pi,-z_{1},-z_{2}\right)$; a diffeomorphism here is provided by the map that sends the equivalence class of $\left(\varphi, z_{1}, z_{2}\right)$ to $\left(\varphi, \zeta_{1}=\right.$ $\left.e^{-i \varphi / 2} z_{1}, \zeta_{2}=e^{-i \varphi / 2} z_{2}\right)$. Note that if $L$ is embedded in $\mathbb{C}^{2}$, then $M$ is embedded in $O(-1) \oplus O(-1)$ and if $L$ is immersed with transverse double points, then $M$ is immersed too. However, the immersion of the latter is not transverse since it is a union of circles.

By the way, this construction is identical to that given by Equation (5.3) of [LMV] when applied to the hyperkähler rotation of the zero locus of a holomorphic function in $\mathbb{C}^{2}$.

Additonal examples come from the construction above in Section 1. In particular, suppose that the braid $\gamma$ has the following property: There exists $k \in\{0, \ldots, N-1\}$ such that

$$
\gamma(\theta+2 \pi(k+1 / 2))=-\gamma(\theta)
$$

at each $\theta \in[0,2 \pi]$. This condition asserts that the braid is mapped to itself by the action of multiplication by -1 on $\mathbb{C}^{2}$. For example, (40) holds when $N$ is odd and $\gamma=e^{i \theta / N}$.

In any event, if $N$ is odd and if (40) holds, then the constructions in Section 1 produce Lagrangians from the braid $\gamma$ that are mapped to themselves by the -1 action on $\mathbb{C}^{2}$. For example, the connect sum of any knot with itself can be represented by a braid with this property.

\section{The view from $T^{*} S^{3}$}

As remarked at the outset, Gopakumar and Vafa came to their conjecture by applying 't Hooft's ideas to certain string theories on $T^{*} S^{3}$. This application suggested a duality between these string theories on $T^{*} S^{3}$ and others on $O(-1) \oplus O(-1)$. As this duality has an explicit geometric basis, the Lagrangians just constructed in $O(-1) \oplus O(-1)$ can be viewed from the perspective of $T^{*} S^{3}$. Such is the purpose of this final section. 
The geometric basis for afore-mentioned duality is simply that both $O(-1) \oplus O(-1)$ and $T^{*} S^{3}$ can be viewed as resolutions of the singularity at the origin in $\mathbb{C}^{4}$ of the zero locus of a certain quadratic polynomial. To be more precise, introduce complex coordinates $\left(c_{1}, \ldots, c_{4}\right)$ for $\mathbb{C}^{4}$. This done, the polynomial in question is

$$
\wp=c_{1} c_{2}-c_{3} c_{4} .
$$

The total space of $O(-1) \oplus O(-1)$ then maps onto $\wp^{-1}(0)$ via a holomorphic map that is one to one off of the zero section and collapses the latter to the origin in $\mathbb{C}^{4}$. The map in question sends the homogeneous coordinates $\left(z, w, \eta_{1}, \eta_{2}\right)$ to

$$
\left(c_{1}=z \eta_{2}, c_{2}=w \eta_{1}, c_{3}=z \eta_{1}, c_{4}=w \eta_{2}\right) .
$$

Meanwhile, $T^{*} S^{3}$ maps to $\wp^{-1}(0)$ as follows: First, take two copies of $\mathbb{R}^{4}$ and use $y \equiv\left(y_{1}, \ldots, y_{4}\right)$ to denote a point in the first and $v \equiv\left(v_{1}, \ldots, v_{4}\right)$ for a point in the second. This done, identify the complement of the zero section in $T^{*} S^{3}$ with the subset of $\mathbb{R}^{4} \times \mathbb{R}^{4}$ where $|y|=|v| \neq 0$ and $\Sigma_{k} y_{k} v_{k}=0$. Here, the convention taken is that the assignment of $|y|^{-1} y \in S^{3}$ to $(y, v)$ defines the projection to $S^{3}$. Now, identify $\mathbb{R}^{4} \times \mathbb{R}^{4}$ with $\mathbb{C}^{4}$ via

- $c_{1}=y_{1}+i v_{1}-i\left(y_{2}+i v_{2}\right)$,

- $c_{2}=y_{1}+i v_{1}+i\left(y_{2}+i v_{2}\right)$,

- $c_{3}=-\left(y_{3}+i v_{3}\right)+i\left(y_{4}+i v_{4}\right)$,

- $c_{4}=y_{3}+i v_{3}+i\left(y_{4}+i v_{4}\right)$.

This map sends the complement of the zero section of $T^{*} S^{3}$ diffeomorphically onto the complement of the origin in $\wp^{-1}(0)$, and it extends in the obvious way as a smooth map from $T^{*} S^{3}$ onto the whole of $\wp^{-1}(0)$ that sends the zero section to the origin in $\mathbb{C}^{4}$.

Now, the preceding describes the 'correspondence diagram'

$$
O(-1) \oplus O(-1) \wp^{-1}(0) \rightarrow T^{*} S^{3},
$$

where both arrows are diffeomorphisms from the complement of the corresponding zero sets to the complement of the origin.

This correspondence gives the following convoluted map from $\left(S^{1} \times\right.$ $\left.\mathbb{C}^{2}\right) /\{ \pm 1\}$ to $\mathbb{C}^{2}$ : First, embed this space in $O(-1) \oplus O(-1)$ by the map 
that sends a point labeled by a unit length complex coordinate $u$ for $S^{1}$ and pair $\left(z_{1}, z_{2}\right)$ of complex coordinates for $\mathbb{C}^{2}$ to the point with the homogeneous coordinates

$$
\left(z=u, w=1, \eta_{1}=2^{-1 / 2} u^{-1 / 2} z_{1}, \eta_{2}=2^{-1 / 2} u^{-1 / 2} z_{2}\right) .
$$

Clearly, the image of this map fibers over the equator in $\mathbb{C P}^{1}$ with fiber $\mathbb{C}^{2}$, and the intersection of the image with the zero section of $O(-1) \times O(-1)$ is the image of $S^{1} \times\{0\}$. Next, use the left arrow in (44) (thus, (42)) to identify the complement of the $S^{1} \times\{0\}$ in $\left(S^{1} \times \mathbb{C}^{2}\right) /\{ \pm 1\}$ with a subset of the complement of the origin in $\wp^{-1}(0) \subset \mathbb{C}^{4}$. This done, use the inverse of the right arrow in (44) (the inverse of (43)) to identify the complement of $S^{1} \times\{0\}$ in $\left(S^{1} \times \mathbb{C}^{2}\right) /\{ \pm 1\}$ with a subset of the complement of the zero section in $T^{*} S^{3}$. Finally, project the latter to $\mathbb{C}^{2}$ using the projection from $T^{*} S^{3}$ to $\mathbb{C}^{2}$ that sends $(y, v)$ to the point with the coordinates $\left(y_{1}+i y_{2}, y_{3}+i y_{4}\right)$. This map extends as a smooth map from $\left(S^{1} \times \mathbb{C}^{2}\right) /\{ \pm 1\}$ to $\mathbb{R}^{4}$ sending $S^{1} \times\{0\}$ to the origin. Amusingly, the map just described is very simple when written with the hyperkähler rotated coordinates $\left(a_{1}, a_{2}\right)$ in (2). Indeed, this map sends $\left(u,\left(a_{1}, a_{2}\right)\right)$ to

$$
2^{-1}\left(u^{-1 / 2} a_{1}, u^{-1 / 2} a_{2}\right) .
$$

The preceding has the following implications: Let $L \subset \mathbb{C}^{2}$ be a Lagrangian surface that is mapped to itself via multiplication on $\mathbb{C}^{2}$ by -1 . Construct from $\mathrm{L}$ the 3-dimensional Lagrangian $M=\left(S^{1} \times L\right) /\{ \pm 1\}$ in $O(-1) \oplus O(-1)$ as described in (39). This done, use the correspondences in (44) to identify the complement of $M$ 's intersection with the zero section with a subset, $M^{*}$, in $T^{*} S^{3}$. Finally, map $M^{*}$ to $\mathbb{C}^{2}$ via the map from $T^{*} S^{3}$ that assigns $\left(y_{1}+i y_{2}, y_{3}+i y_{4}\right)$ to $(y, v)$.

The result is a smooth map from $\left(S^{1} \times L\right) /\{ \pm 1\}$ to $\mathbb{C}^{2}$ that simply rotates $L$ as in (46). In particular, if $L$ intersects some 3 -sphere about the origin as a knot, then for each fixed $u \in S^{1}$, the corresponding image of $(u \times L)$ in $\mathbb{C}^{2}$ intersects the concentric half radius sphere as a rotated image of the same knot.

\section{References}

[AKV] M. Aganagic, A. Klemm, A. and C. Vafa, Disk instantons, mirror symmetry and the duality web, preprint 2001.

[F] A. Floer, Morse theory for Lagrangian intersections, J. Diff. Geom., 28 (1988), 513-547. 
[FP] C. Faber and R. Pandharipande, Hodge integrals and Gromov-Witten theory, Invent. Math., 139 (2000), 173-199.

[G] M. Gromov, Partial Differential Relations, Springer-Verlag, 1986.

[GV] R. Gopakumar and C. Vafa, On the gauge theory/geometry correspondence, Adv. Theor. Math. Phys., 3 (1999), 1415-1443.

$[\mathrm{H}] \quad$ G. 't Hooft, A planar diagram theory for strong interactions, Nucl. Phys., B72 (1974), 461-473.

[KL] S. Katz and C.-C. Liu, Enumerative geometry of stable maps with Lagrangian boundary conditions and multiple covers of the disk, preprint, 2001.

[LM] J.M.F. Labastida and M. Marino, Polynomial invariants for torus knots and topological strings, Commun. Math. Phys., 217 (2001), 423-449.

[LMV] J.M.F. Labastida, M. Marino and C. Vafa, Knots, links and branes at large $N$, J. High Energy Phys., 11(7) (2000), 42 pp.

[OV] H. Ooguri and C. Vafa, Knot invariants and topological strings, Nucl. Phys., B577 (2000), 68-108.

[RS] P. Ramadevi and T. Sarkar, On link invariants and topological string amplitudes, preprint, 2000.

[LS] J. Li and Y.S. Song, Open string instantons and relative stable morphisms, preprint, 2001.

[W] E. Witten, Chern-Simons gauge theory as a string theory, in 'The Floer Memorial Volume', H. Hofer, C.H. Taubes and A. Weinstein, eds., Birkhäuser, (1995), 637-678. 